\title{
Jean-Paul Durand
}

\section{A propos du mariage homosexuel? Droit, éthique, théologie}

Prawo Kanoniczne : kwartalnik prawno-historyczny 50/3-4, 89-107

2007

Artykuł został zdigitalizowany i opracowany do udostępnienia w internecie przez Muzeum Historii Polski w ramach prac podejmowanych na rzecz zapewnienia otwartego, powszechnego i trwałego dostępu do polskiego dorobku naukowego i kulturalnego. Artykuł jest umieszczony w kolekcji cyfrowej bazhum.muzhp.pl, gromadzącej zawartość polskich czasopism humanistycznych i społecznych.

Tekst jest udostępniony do wykorzystania w ramach dozwolonego użytku. 
JEAN-PAUL DURAND

L'Institut Catholique de Paris

\section{A PROPOS DU MARIAGE HOMOSEXUEL? DROIT, ÉTHIQUE, THÉOLOGIE}

Plan: 1. Lacces hétérosexuel au mariage civil. 1.1. Il importe de parler de la nullité juridique du mariage homosexuél. 1.1.1. En droit positif francais. 1.1.2. Qu'en est-il droit européen. 1.2. Instaurations de quelques succédanés du mariage. 2. Compagnonnage, couple, mariage. 2.1. A propos de la notion de «couple homosexuel». 2.2. A propos de la notion de mariage en Europe.

Droit, éthique, théologie? Ne faudrait-il pas inverser l'ordre de ces disciplines, afin d'éviter une démarche risquant de souffrir de positivisme? J'ai essayé ici d'identifier l'un des défis méritant d'être considéré à nouveaux frais, à savoir le formalisme consistant à exiger que le droit en vigueur se contente de suivre l'évolution des mours. La tache des législateurs ne tend-elle pas à se rapprocher de l'ethos, plus que des théologies? Quant à l'éthique, un tel registre n'est-il pas tiraillé de toutes parts? Or, le droit des principes fondamentaux ne peut-il pas devenir un précieux allié de l'éthique, cette dernière pouvant revenir à ce qu'elle est: exigence d'humanité, médiation humanisante, attention à l'ethos et ouverture transcendantale, délibération de la conscience éclairée à propos de la décision et de l'indécidable.

Le droit privé francais s'est formé au cours d'une histoire plus que bimillénaire. Les notions et les règles concernant le mariage, la filiation, les régimes matrimoniaux, les successions, la propriété, les contrats et la responsabilité, s'enracinent dans le droit romain et se sont ensuite enrichies de l'apport des Eglises chrétiennes, des coutumes, de réflexions doctrinales, de la législation royale puis révolutionnaire, mais aussi des crises ultérieures de modernité et de post-modernité. Traditions nombreuses entre lesquelles le Code 
civil de 1804' s'est efforcé, dans un souci d'unité sans doute, d'établir une sorte de transaction. Les évolutions n'ont pas manqué, mais existe-t-il une définition de la famille en droit positif francais? Pour quelle raison, est-il si difficile d'en préciser une catégorie juridique? Comment la société francaise s'engage-t-elle à propos du lien conjugal ${ }^{2}$ et du lien parental ${ }^{3}$ ? Qu'est-ce qui retient en premier l'attention en cette fin d'hiver de l'année 2007? Est-ce le contrat? Est-ce que ce sont les dimensions du lien qui retiennent l'attention et forcent le respect? Le sentiment a pris sa place dans le mariage. Quant à la parole donnée par l'échange des consentements, ne constitue-t-elle pas un relais indispensable, y compris pour parer à tel ou tel risque de subjectivisme? Ce dernier trait de pensée n'a-t-il pas cessé de croitre en bien et en mal depuis le XVI ${ }^{\mathrm{e}}$ siècle dans nos mentalités occidentales?

Tout à tour j'évoquerai les enjeux institutionnels de l'accès hétérosexuel au mariage civil. Dans ce cadre, je noterai combien le mariage homosexuel n'a pas encore de statut juridique dominant en France, ni dans la plupart des autres dimensions de l'Europe, pour ne parler que de ces régions dont je suis plus familier (I).

Puis, j'évoquerai brièvement quelques enjeux sémantiques de nature juridique: qualifier juridiquement les partenariats, les couples et le mariage civil, cela ne manque pas de nourrir aussi la réflexion éthique et théologique. Je ne traiterai pas des mariage religieux, ni spécialement du mariage entre baptisés, ce qui demanderait de nouveaux exposés ${ }^{4}$. Je me suis appliqué à rester attentif

\footnotetext{
'Ministère de la Justice, "Vivre et faire vivre le Code civil», Bicentenaire du Code civil organisé par la Chancellerie au Palais du Luxembourg, le 29 avril 2004, in Les Petites affiches, $\mathrm{N}^{\circ} 216$, octobre 2004, 72 pages; Jean-Francois NIORT, Homo civilis, contribution à l'histoire du Code civil francais (1804-1965), Préface de Jean-Louis Halpérin, postface de Jean Carbonnier (+), Aix-en-Provence, Presses universitaires d'Aix-Marseille, 2004, 2 tomes, 931 pages.

${ }^{2}$ D. Fenouillet, «Le lien conjugal», publié par D. Mazeaud (dir.), La personne dans tous ses Etats, in Les Petites affiches, $\mathrm{N}^{\circ} 131$, juillet 2004, p. 58-69.

${ }^{3}$ F. Dkeuwer-Défossez, «Le lien parental», Ibid., p. 70-75.

${ }^{4} \mathrm{Cf}$. notamment Xavier Lacroix, L'avenir, c'est l'autre, dix conférences sur l'amour et la famille, collection Recherches morales, Paris, Cerf, 2001, 235 pages: Paul Moreau, La famille, enjeu citoyen, collection Recherches morales, Paris, Cerf, 2002, 210 pages; Xavier Lacroix (dir.), Oser dire le mariage indissoluble, collection Recherches morales, Paris, Cerf, 2001, 243 pages; L. Bonnet, La communauté de vie conjugale au regard des lois de l'Eglise catholique, postface de Gaston Candelier, collection Droit canonique, Paris. Cerf, 2004, 532 pages; Gaston Candelier, Le droit de l'Eglise au senice des épotux.
} 
aux évolutions du droit civil positif, tant en France, qu'aux échelons de la construction européenne (II). En conclusion, j'évoquerai le délicat problème de la revendication d'un droit à l'adoption dans le cadre d'un compagnonnage homosexuel, ce qui soulève la difficulté de raisonner en termes d' "homoparentalité» pour le droit civil, l'éthique et en théologie.

\section{L'accès hétérosexuel au mariage civil}

Est-il possible, dans un article, de donner une information juste sur la tendance générale ou moyenne des législations en France et en Europe, quant à leur attitude concernant les conditions du mariage civil liées à la différence de sexe des conjoints? En tout cas, il faut mentionner la revendication en faveur d'un mariage homosexuel, que certains lobbies voudraient ériger en une alternative matrimoniale: quel est le traitement juridique de la revendication pour le mariage homosexuel? Quel traitement est appliqué par le droit positif aux quelques mises en pratique de ce principe revendiqué?

\subsection{Il importe de parler de la nullité juridique du mariage homosexuel}

\subsubsection{En droit positif francais}

Qu'en est-il du Code civil?

Dès l'origine, le Code civil francais n'énonce pas expressément la différence de sexe comme condition du mariage, car cette différence allait de soi pour les rédacteurs du Code civil en 1804. Les références faites à l'homme et à la femme, comme étant unis par les liens du mariage, étaient cependant nombreuses dans les travaux préparatoires. Ce n'est que dans le contexte principiel de l'égalité entre chacun des époux, dans le cadre de cette institution matrimoniale, que les mots «homme» et «femme» ont fait la place au mot «époux». La différence de sexe est encore présente dans le Code civil: il faut se référer à l'article 144 non modifié depuis 1804 , mais aussi à l'article 75, un article qui impose aux deux parties, lors de la célébration, de déclarer qu'elles veulent se prendre pour mari

préface de Jacques Gressier, collection Droit canonique, Paris, Cerf, 1999, 195 pages; Yves-Marie Blanchard (dir.), «Léchec du mariage des chrétiens: réponses canoniques et pastorales dans diverses confessions», in Reviue d'éthique et de théologie morale «Le Supplément», $\mathrm{N}^{\circ} 228$, mars 2004 , p. 240-308. 
et femme. Une référence à l'homme et à la femme est faite aussi dans les textes concernant la communauté de vie et le domicile des époux (article 108 du Code civil), de même que dans les articles 264 et 300 du même Code qui régissent les conséquences du divorce ou de la séparation de corps.

Pour le législateur francais, le PACS ("pacte civil de solidarité») n'est pas un mariage.

Selon les travaux préparatoires de la loi $\mathrm{N}^{\circ}$ 99-944 du 15 novembre 1999 concernant le pacte civil de solidarité, il ressort que dans l'esprit du législateur, il y avait lieu de donner par le moyen du PACS une base juridique au compagnonnage homosexuel, étant donné que pour le même législateur, le mariage civil est réservé à l'union d'un homme et d'une femme. Je reviendrai plus loin sur ce régime juridique nouveau et encore fragile.

En droit positif francais, la différence des sexes est donc une condition du mariage civil.

La culture francaise est encore largement attachée à cette condition que pose la législation francaise, en faveur de l'exigence d'une différence de sexe pour contracter mariage.

Certes, les mentalités évoluent: en 1975, 24\% seulement des personnes interrogées en France estimaient que l'homosexualité était une manière acceptable de vivre sa sexualité. En 1996, la proportion est montée à $67 \%$, selon le rapport remis à l'Assemble nationale en 1998 par M. Michel's. Le législateur doit-il suivre toutes les évolutions ou résister au contraire à certaines mutations, comme ici pour préserver le couple hétérosexuel et préserver par conséquent la famille, dont celui-ci est le fondement constitutif?

Le Pape Jean Paul II à cet égard n'interpelle pas seulement la conscience de chaque catholique et la conscience de toute personne de bonne volonté. Le regretté Pape n'avait pas hésité à s'adresser aux Etats et organisations internationales, en ce qui concerne leur responsabilité pour le bien personnel de chaque individu et le bien commun de la condition humaine, afin que la loi ne se contente pas de céder aux opinions pratiques qui peuvent devenir majoritaires ${ }^{6}$.

\footnotetext{
${ }^{5}$ Rapport $\mathrm{N}^{\circ} 1097 \mathrm{du} 23$ septembre 1998.

' Jean Paul II, L'Evangile de la vie (Evangelium vitae), collection Documents des Eglises, Paris, Cerf, 1995, 192 pages.
} 
Deux motifs formels sont en effet agités pour exiger que le droit positif légalise les mariages homosexuels ou lesbiens: d'une part, l'évolution des mœurs et d'autre part les conditions quant au respect d'un principe d'égalité. Egalité, si les mentalités en viennent à considérer le point de vue culturel d'après lequel, à côté de la condition hétérosexuelle, existerait une «condition homosexuelle». En d'autres termes, qu'il n'existerait pas seulement des personnes ayant des tendances et/ou des pratiques homosexuelles, lesbiennes ou bisexuelles. La civilisation serait-elle en présence d'une «condition homosexuelle»? Personnellement, je ne le pense pas.

Dans cette affaire, la composante culturelle et les lobbies financiers occupent une importance considérable. Pour sa part, le débat scientifique est loin d'être unanime pour valider ce phénomène d'homosexualité. en termes d'alternative au phénomène hétérosexuel. Consultons les propos tenus lors de la préparation du débat parlementaire - qui ont conduit au vote de la loi francaise du 31 décembre 2004 «portant création de la haute autorité de lutte contre les discriminations et pour l'égalité»" ${ }^{7}$-: ces propos montrent heureusement que la liberté de la critique est encore sauve en France pour tout un chacun. Ainsi par exemple, journalistes, scientifiques, éthiciens et théologiens, notamment, ont donc encore en France la liberté de conclure à l'existence non fiable d'une condition homosexuelle alternative à la condition hétérosexuelle en matière de conjugalité par excellence. Qu'ils aient la liberté de continuer de s'exprimer sur leur scepticisme à l'égard de certains combats menés par des lobbies "gay». Ces manifestations «gay», beaucoup de journalistes et sociologues n'hésitent pas à les qualifier de modes culturelles.

${ }^{7}$ Loi $\mathrm{N}^{\circ} 2004-1486$, in $J O \mathrm{~N}^{\circ} 304$ du 31 décembre 2004, p. 22567; le rapporteur du Sénat, M. Hyest a clairement dit, en première et deuxième lectures, que l'on continuerait par exemple à pouvoir mentionner en France les passages de la Bible qui condamnent cette pratique de l'homosexualité en termes particulièrement virulents, (cf. le livre de la Genèse et des enseignements de l'apôtre saint Paul). Le Garde des Sceaux et ministre de la Justice, $M$. Dominique Perben, a rappelé le maintien de la liberté d'expression critique. Les ministres présents à cette procédure législative ont été attentifs au nom du Gouvernement à cette liberté critique à laquelle les journalistes en particulier sont attachés. Il faut citer Mme Nelly Olin, ministre déléguée à l'Intégration, à l'Egalité des chances et à la Lutte contre l'exclusion, ainsi que Mme Nicole Ameline, ministre de la Parité et de l'Egalité professionnelle (Projet de loi de la Halde, voté fin décembre 2004). 
Comportements et opinions critiques de chaque personne doivent être respectés, du moment qu'en démocratie, les limites de l'ordre public ne sont pas dépassées.

Or cet ordre public comprend désormais le principe juridique de non discrimination pour motif d'orientation sexuelle. La vie sociale en France et en Europe est en charge de ce double défi, entre d'une part le droit positif et l'ordre public qui permettent la liberté de l'orientation sexuelle, et d'autre part le débat critique sur l'évaluation de la valeur des comportements, ici sexuels, au regard de l'anthropologie, de l'éthique, en théologie et aussi selon le droit en vigueur dans le pays.

Le droit positif, sans aucun doute, doit protéger tous les humains: individus et personnes, hommes et femmes, jeunes et vieux, de toute race, quelque soient leurs convictions et appartenances religieuses, et donc aussi sans admettre la moindre discrimination pour motif d'orientation sexuelle.

Mais une chose est de respecter les personnes ayant des tendances et/ou des pratiques homosexuelles, autre chose est d'ajouter à ces orientations sexuelles, au moyen du droit positif, des valeurs qui correspondent par nature à d'autres modalités: celles qui sont communes et inhérentes à une existence humaine vécue en couples hétérosexuels, à la transmission de la vie, à la fondation de familles et à l'éducation des enfants.

Certes, les cultures et les traditions sexuelles, matrimoniales ont varié considérablement dans l'histoire de l'humanité; elles restent souvent fort diverses ${ }^{8}$. Mais les valeurs les plus fondamentales sont étalonnées par les grandes religions, par la personne du Christ, par son Eglise, et cela, malgré de profonds débats dans et entre certaines Eglises: accès plus sévères à l'état de vie consacrée, à la cléricature; controverses sur l'accès aux ministères et vives controverses lors d'un accès à l'épiscopat.

La moralité est accessible à toute conscience humaine, conscience que la Trinité sainte éclaire. Dieu révélé en Jésus Christ apporte le sens tant fondateur, qu'ultime de la moralité: existe en effet une finalité, mais à la condition qu'elle se libère des utopies. Il en va

${ }^{8}$ Collectif, Sexualité humaine, histoire, ethnologie, sociologie, psychanalyse, philosophie (centre d'étude Laennec), Paris, Lethielleux, 1966, 339 pages; Maurice Godelier, Métamorphoses de la parenté, Paris, Fayard, 2004. 
des conditions du salut eschatologique de chaque créature, le bien commun devant en comporter un souci constant.

Cela étant rappelé, comment vont évoluer les mentalités? Quelles seront demain les approches du mariage, de la conjugalité, de la parentalité? Quelle sera l'intervention du droit en vigueur? Selon le jugement en date du 27 juillet 2004 du Tribunal de grande instance de Bordeaux ${ }^{9}$ la différence des sexes est encore en droit francais une condition du mariage. Il en résulte que l'union contractée entre deux personnes de même sexe doit être annulée, cette union ne respectant pas les conditions de la différence des sexes pour toute union matrimoniale. Ainsi avait été annulé le mariage homosexuel célébré à Bègles en Gironde. Trois mois après, la mairie de Bagnolet dans la Seine-Saint-Denis a publié les bans d'un mariage entre deux hommes. La municipalité dirigée par M. Marc Everbecq, communiste, a affiché la nouvelle sur les panneaux de l'Hôtel de ville: le journal catholique La Croix rédigea un «bref» le 8 septembre 2004 à ce propos (p. 8).

\subsubsection{Qu'en est-il en droit européen?}

Les normes européennes connaissant un processus d'homogénéisation dans le contexte de l'intégration européenne et de la construction de l'Union européenne ${ }^{10}$.

Selon la jurisprudence de la Cour européenne des droits de l'homme, siégeant à Strasbourg, en vertu de l'article 12 de la Convention européenne de sauvegarde des droits de l'homme et des libertés fondamentales, signée à Rome en 1950: «à partir de l'age nubile, l'homme et la femme ont le droit de se marier et de fonder une famille selon les lois nationales régissant l'exercice de ce droit». La Convention de 1950 a voulu protéger le droit au mariage entre deux personnes de sexe différent. La jurisprudence de la Cour européenne de Strasbourg dans son arrêt Goodwin du 11 juillet

\footnotetext{
${ }^{9}$ Première chambre civile, in Recueil Dalloz, $\mathrm{N}^{\circ} 33,2004$, p. 2392-2393, note d'Eric Agostini, p. 2393-2395.

${ }^{10}$ On sait que chacun des vingt-cinq membres de l'Union européenne adhère à la Convention européenne de 1950 et que le Traité constitutionnel pour l'Union européenne de 2004, actuellement en cours de réception par chaque Etat membre, assume la Convention européenne de sauvegarde des droits de l'homme; ce qui donne aux différentes normes de la construction européenne une cohésion jusqu'alors jamais atteinte à ce point.
} 
$2002^{11}$ a réaffirmé dans des conditions complexes - mais par décision prise à l'unanimité des juges -, ce principe de l'hétérogénéité inhérent au mariage. La Cour a admis en l'espèce le principe du droit au mariage concernant une personne ayant subi une opération de conversion sexuelle (transsexualisme) et qui désirait épouser une personne ayant le même sexe qu'elle, du point de vue de l'état civil. La Cour réaffirme en effet implicitement le principe d'hétérosexualité du mariage: elle précisait qu'il ne fallait pas ignorer le changement de sexe de la personne dans le domaine corporel depuis cette intervention chirurgicale.

Dans le même sens, le 7 janvier 2004, le Cour de justice des Communautés européennes, siégeant à Luxembourg, réaffirme à propos d'un cas de transsexualisme, le principe de la différence de sexes pour contracter un mariage ${ }^{12}$.

"Cour européenne des droits de l'homme, 11 juillet 2002, C. Goodwin et I. c/ Royaume-Uni, non publiée, points 97 à 104; in Dalloz, 2003, jur. P. 2032, note A.-S. Chavent-Leclère.

${ }^{12}$ Voir: CJCE, C-117-01: demandeur: K. B. -Défendeur: National Health Service Pensions Agency, 7 janvier 2004, site www.dalloz.fr et CD-Rom du Recueil Dalloz.; Philippe Icard, «Note. Un droit d'accès au mariage protégé par la CJCE», in Recueil Dalloz, $\mathrm{N}^{\circ} 14,2004$, p. 979-983: «Sommaire de la décision: Larticle $141 \mathrm{CE}$ s'oppose, en principe, à une législation qui, en violation de la Convention européenne de sauvegarde des droits de l'homme et des libertés fondamentales, signée à Rome le 4 novembre 1950, empêche un couple, composé d'une femme et d'un homme transsexuel né de sexe féminin et enregistré comme tel à létat civil (en Grande Bretagne) qui, à la suite d'une opération médicale de changement de sexe, est devenu un homme, de remplir la condition de mariage nécessaire pour que l'un d'entre eux puisse bénéficier d'un élément de la rémunération de l'autre. Il incombe au juge national de vérifier si, dans un tel cas, une personne peut se fonder sur l'article $141 \mathrm{CE}$, afin de se voir reconnaitre le droit de faire bénéficier son partenaire d'une pension de réversion». (p. 979). Philippe Icard a introduit sa note par cette question: «Linterdiction par une législation de modifier l'état civil d'une personne du fait d'un changement de sexe, afin qu'elle puisse se marier, constitue-t-elle une discrimination au vu du droit communautaire?». En Grande Bretagne, le mariage entre homosexuels n'est pas reconnu. Du point de vue juridique, le droit britannique ne permet pas de modifier l'acte de naissance. En réalité, il ressort de cet arrêt de la CJCE que le refus de mariage n'est pas la conséquence directe du changement de sexe. Ce refus a pour origine l'impossibilité pour un transsexuel de modifier son acte de naissance. En droit britannique, c'est toujours un compagnonnage homosexuel. Il s'en suite qu'il ne revient pas à la CJCE d'exiger d'un Etat que ce dernier assimile «vie en couple et mariage.» Lélément décisif quant à l'inégalité consiste finalement à réduire l'accès au mariage, en ayant exclu la modification de l'état civil même après l'opération médicale de changement physique de sexe. Cette rigueur civile britannique entraine une non prise en compte d'un principe général du droit communautaire, la CJCE reconnaissant que les transsexuels joutissent d'un droit fondamental à contracter mariage dans les conditions prenant en considération leur nouveau sexe. La CJCE affir- 
Certes, l'article 8 de la Convention européenne de 1950 garantit le droit de toute personne au respect de sa vie privée et familiale. Mais la jurisprudence et la doctrine concluent que la limitation du droit au mariage aux personnes de sexe opposé ne constitue pas une atteinte à ces principes. La jurisprudence a pourtant posé le principe que toute discrimination est interdite, quel que soit le motif qui la fonde. Selon l'article 14 de la Convention européenne de sauvegarde des droits de l'homme et des libertés fondamentales de 1950, la jouissance des droits et libertés reconnus dans la présente convention doit être assurée, sans distinction aucune, y compris de sexe.

La condition exigeant la différence de sexe pour conclure un mariage est-elle constitutive d'une discrimination au sens de cet article 14? La jurisprudence a aussi affirmé qu'il n'y avait pas de discrimination en l'absence de situation identique, ou qui s'en rapproche. A première vue, il existe une différence entre deux réponses de la Cour européenne des droits de l'homme siégeant à Strasbourg: d'un côté, le principe de l'interdiction du mariage à un compagnonnage entre deux personnes de même sexe et d'un autre côté le principe de la liberté de choix de l'orientation sexuelle. Mais pour la Cour européenne des droits de l'homme, cette différence est acceptable et possible: il suffit d'une part qu'existe une justification objective et raisonnable poursuivant un but légitime dans une société démocratique; et il suffit d'autre part, que ce motif respecte, entre les moyens et le but, un rapport de proportionnalité raisonnable. Or c'est le cas, estime la Cour, pour le mariage qui est une institution traditionnelle considérée très largement comme étant à l'origine de la fondation de la famille.

Ce consensus est en partie seulement celui des Etats européens: le mariage est propre à l'union d'un homme et d'une femme qui veulent fonder une famille.

Fin 2004, déjà deux Etats européens avaient modifié leur législation en faveur du mariage homosexuel: la Hollande le 21 décembre

me que «la décision de réserver certains avantages aux couples mariés, en excluant tous ceux qui cohabitent sans être mariés, relève soit du choix du législateur, soit de l'interprétation effectuée par les juridictions nationales des règles juridiques de droit interne, sans que les particuliers puissent faire valoir aucune discrimination fondé sur le sexe, interdite par le droit communautaire» (Point 28, p. 981). 
2000 et la Belgique le 13 février 2003. L'Espagne ${ }^{13}$ a pris ce chemin; le Congrès des députés votant une loi pour la mariage homosexuel en juin 2005. Quant au Royaume Uni, il a légalisé le pacte civil («Civil partnership») à partir du 5 décembre 2005.

Entre temps, le 20 juillet 2005 , le Canada rend légal ce type de mariage, qui devient valide en Afrique du Sud depuis le vote de la première loi favorable à ce principe en Afrique, le 14 novembre 2006.

Revenons à l'Europe: En Allemagne et en Suède, il est question de changer la législation nationale. En France, aucune loi n'a modifié le principe de la différence de sexe pour contracter mariage.

La Cour européenne des droits de l'homme n'a pas sanctionné les législations nationales exigeant une différence de sexe entre les époux. Le 8 juillet 2002, la Cour avait jugé que l'union contractée entre deux personnes, si elle ne remplissait pas les conditions du mariage au regard de cette différence de sexe, devait être annulée $e^{14}$.

\subsection{Instaurations de quelques succédanés du mariage}

Des législations d'autres pays que la France ont décidé, ou ont envisagé, de donner un statut aux compagnonnages homosexuels, mais pas toujours pour les autoriser à conclure un mariage. On assiste ainsi à la mise en place de succédanés du mariage, comme le PACS en France ${ }^{15}$.

A cela s'ajoute une autre réalité dont il faut considérer l'importance et qui peut exprimer un malaise à l'encontre de l'institution

\footnotetext{
${ }^{13}$ En novembre 2004, lorsque cette conférence a été donné, le Gouvernement socialiste de M. José Luis Rodriguez Zapatero avait présenté son avant-projet de loi le $1^{\text {er }}$ octobre précédent, et il comptait faire adopter par les Cortès son texte d'autorisation des mariages homosexuels pour janvier 2005. La chose est faite.

${ }^{14}$ Cour européenne des droits de l'homme, 8 juillet 2002, Somm. P. 252, obs. C. Birsan; Ibid., p. 1935, obs. J.-J. Lemouland V; et voir note in Recueil Dalloz, N ${ }^{\circ} 31$, 2004, p. 2270-2271 (décision in extenso sur Dalloz actualité: www.dalloz.fr); P. Malaurie, «La dignité de la personne humaine, la liberté sexuelle et la Cour européenne des droits de l'homme ", in Petites affiches, $\mathrm{N}^{\circ} 152,1^{\circ}$ aoit 2006, p. 6-7.

${ }^{15} \mathrm{~J}$.-L. Vivier, Le pacte civil de solidarité, un nouveau contrat, collection Logiques juridiques, Paris, LHarmattan, 2001, 184 pages; Y. Delecraz, «Le régime patrimonial du PACS» in Actualité juridique. famille, N7-8, 2004, p. 269-271; G. Kessler, «La reconnaissance en France des partenariats enregistrés à l'étranger", in Actualité juridique. famille, N`7-8, 2004, p. 272-277; X. Labbée, «PACS: Quelles finalités? Quelle réforme?», in Actualité juridique. famille, $\mathrm{N}^{\circ} 7-8,2004$, p. 256-260; H; Fulchiron, «Quel avenir pour le PACS?», in Répertoire du notariat Defrénois, 15 septembre 2005, N¹7, p. 1286.
} 
du mariage: ces formules mises à la disposition de personnes homosexuelles sont utilisées aussi par des hétérosexuels qui vivaient déjà en concubinage et qui ne sont pas parvenus à choisir l'institution du mariage. En Italie, le projet très contesté du Gouvernement de M. Prodi du 9 février 2007 en faveur du «Dico» (Droits des personnes vivant ensemble) a visé des liens non matrimoniaux et non uniquement entre personnes de même sexe.

Je ne développe pas ici le dossier francais du PACS j'attire seulement l'attention sur sa fragilité, mais il se développe en nombre d'usagers (voir Annexe in fine). Des mesures sont déjà venues en affermir le régime. Je relèverai une limite inquiétante: la cessation du PACS ne peut-elle pas ressembler à une sorte de répudiation lorsqu'elle n'est pas assumée de commun accord?

En face de différents succédanés au mariage civil, il y a notamment le mariage-sacrement: cette institution, elle, est beaucoup plus que le désir, plus que le sentiment; la volonté et le consensualisme y ont une décisive, mais toujours en étant précédés par le Grace divine. Car le bien naturel conjugal est élevé à la capacité de rapprocher l'humanité de Dieu, Dieu révélé en Jésus Christ. Et cette capacité est donnée gratuitement par Dieu lui-même. Si Dieu a pu donner librement son amour, il ne reprend pas ce qu'il a donné. Quant aux conjoints chrétiens, ils sont invités à se nourrir de cette antécédence divine gratuite, aimante, et pleine de compassion.

La vie affective, les liens familiaux, la conjugalité, sont aujourd'hui marqués par nombre d'incertitudes et d'hésitations, même si subsistent des traditions nationales ou régionales. Quant à la construction européenne et à certains effets de la mondialisation, ils peuvent non pas seulement ouvrir les esprit et les cours, mais aussi parfois, hélas, imposer des formalismes susceptibles de nier le rapport humain à l'autre.

\section{Compagnonnage, couple, mariage}

Autour de l'identité sexuelle, autour du corps, autour de l'éducation, des domaines de flou semblent s'étendre. Il s'agit d'être attentif aux avancées d'une culture de la liberté et du désir.

Cette culture génère aussi son contraire en quelque sorte: ne faut-il établir aucun lien entre cette modernité et ces différentes formes d'attachement très traditionalistes, par exemple, à certains 
usages des plus strict en ce qui concerne le statut des femmes: voile islamique, difficultés de renoncer dans la collectivité francaise de Mayotte, dans l'Océan Pacifique, à la répudiation et à la polygamie, ou encore à, en plein, cour des métropoles occidentales, fort l'attachement à des décisions traditionnelle autoritaires et machistes en ce qui concerne des épousailles? Ainsi cohabitent difficilement avec l'ordre public libéral, des formes d'ascétisme extrêmes ou d'intégrisme: ces formes identitaires restent non seulement encore vivaces, mais elles connaissent parfois des poussées de virulence, d'intolérance, de revendications exigeant l'instauration d'un communautarisme constitué à la fois de fermeture et de discipline portant atteinte aux droits individus, aux droits des couples, sous le poids de réseaux ou de maillages communautaires autoritaires.

Certes, la subjectivité immédiate ou les aléas de l'affectivité ne peuvent pas être les seules références de la conduite de la vie relationnelle. Qu'en est-il de l'éthique et du droit de la communauté et de la responsabilité? Qui dit communauté ne dit pas toujours communautarisme abusif. Qui dit responsabilité, ne dit pas toujours culpabilisation excessive.

\subsection{A propos de la notion de «couple homosexuel»}

En France, il existe une reconnaissance sociale et juridique du compagnonnage homosexuel en terme de «couple». Il suffit de se reporter désormais à l'article 515-8 du Code civil qui définit le concubinage comme étant l'union entre deux personnes de sexe différent ou de même sexe, vivant en couple.

Cette évolution n'a-t-elle pas été préconisée par la résolution du Parlement des communautés européennes le 8 février 1994, qui encourage les Etats membres d'œuvrer en faveur de l'égalité des droits? Une chose est l'égalité des sexes, autre chose est l'indifférenciation sexuelle.

Une validation civile risquant de tendre à une admission du principe de l'indifférenciation sexuelle ne fait pas obligation aux éthiciens, théologiens et scientifiques de changer d'opinion, si leurs convictions respectives les portent à estimer toujours que la notion de couple homosexuel risque d'entrainer des confusions en anthropologie sociale.

Par ailleurs, il ne s'agit pas non plus d'idéaliser la condition hétérosexuelle: la condition hétérosexuelle n'est pas pour autant 
considérée comme si elle était exemptée de faiblesses humaines, ni de déchirements sociaux, ni de diversités culturelles. Mais les séparations et divorces que connaissent les couples hétérosexuels, ne pourraient-ils pas être compris comme étant, pour une part, les résultats de mentalités cultivant l'indifférenciation des sexes, la subjectivisation des identités, celle des genres, et une crise d'identification des rôles respectifs de père, de mère, d'enfants ${ }^{16}$ ?

Même, je le disais plus haut, des Eglises chrétiennes protestantes et anglicanes, chez des pays voisins de la France, aux Etats-Unis ou en Afrique, sont traversées par de rudes débats concernant l'homosexualité et les conséquences à en déduire en pastorale et quant aux critères d'idonéité pour qu'une Eglise appelle tels fidèles aux ministères ${ }^{17}$.

${ }^{16}$ Jean-Marie Ghitti, La séparation des familles, collection Recherches morales, Paris, Cerf, 2003, 157 pages; Id., L'Etat et les liens familiaux. Mécanismes de la domination, collection Recherches morales, Paris, Cerf, 2004, 153 pages; Id., Pour une éthique parentale, essai sur la parentalité contemporaine, collection Recherches morales, Paris, Cerf, à paraitre en 2005; autres point de vue: $\mathrm{H}$. Fulchiron, «Mariage et partenariats homosexuels en droit international privé francais», in Revue internationale de droit comparé, 2, 2006, p. 409-438; du même auteur, "Le droit francais et les mariages homosexuels étrangers», in Recueil Dalloz, $\mathrm{N}^{\circ} 19,2006$, p. 1253-1258; et son étude à propos de l'arrêt de la $1^{\circ}$ chambre civile du 24 février 2006: «Parenté, parentalité, homosexualité», in Recueil Dalloz, N¹3, 2006, p. 876-877.

${ }^{17}$ S'agissant de l'homosexualité: la Commission internationale anglicane-catholique (ARCIC II) a achevé sa tache en mai 1999 en publiant notamment ce texte sous le titre «Accords et désaccords» (extrait)... «N $N^{\circ} 87$. Nous avons une situation similaire en matière de relations homosexuelles. Nos deux Communions affirment toutes deux l'importance et la signification de l'amitié et de l'affection entre hommes et ente femmes, mariés ou célibataires. Toutes deux affirment que toutes les personnes, y compris celles à tendances homosexuelles, sont faites à l'image de Dieu et partagent la pleine dignité qui leur vient du fait d'ętre des créatures humaines. Toutes deux affirment qu'une union conjugale fidèle et pour la vie entre un homme et une femme, offre le contexte normatif pour une relation sexuelle pleinement accomplie. Toutes deux se réfèrent aux Ecritures et à l'ordre naturel en tant que sources de leur doctrine dans de domaine. C'est pourquoi elles rejettent toutes deux l'assertion avancée parfois, selon laquelle les rapports homosexuels et conjugaux sont moralement équivalents et également capables d'exprimer l'ordre et l'usage correct des pulsions sexuelles. Un tel ordre et un tel usage sont, croyons-nous, un aspect essentiel de la vie en Christ. Dans ce cas également, nos manières différentes de formuler la loi sont significatives (cf. $\mathrm{N}^{\circ} 52$ ). La doctrine catholique affirme que l'activité homosexuelle est 'intrinsèquement désordonnée' et conclut qu'elle est toujours objectivement un mal. Cette attitude se reflète dans le genre d'assistance pastorale apportée aux homosexuels. Les anglicans peuvent admettre qu'une telle activité est déréglée, mais on peut très bien trouver entre eux des différences quant à l'assistan- 
En tout cas, il existe une différence de traitement en droit francais pour des personnes de même sexe en ce qui concerne d'un côté les conditions du concubinage et celles du PACS, et d'un autre côté les conditions de l'accès au mariage.

A propos de cette résolution du 8 février 1994 du Parlement européen, celle-ci préconisait des contrats d'union civils, non des extensions, hors de la différenciation sexuelle, de l'image du mariage; une image déjà si diversifiée à travers les cultures dans l'histoire des civilisations et aujourd'hui encore à travers les différents espaces et bassins culturels de notre planète Terre.

\subsection{A propos de la notion de mariage en Europe}

En droit européen, qu'en est-il? Je n'avancerai tout au plus que quelques remarques. Malgré la très difficile réception du Traité constitutionnel de l'Union européenne depuis 2004, traité comportant la Charte des droits fondamentaux adoptée à Nice en 2000, l'intégration européenne devrait concerner aussi le droit des personnes d'une certaine manière.

Cependant, chaque Etat membre peut affirmer une autonomie compatible avec la Convention européenne de sauvegarde des droits de l'homme et des libertés fondamentales signée en 1950 et compatible avec son importante jurisprudence.

ce morale et pastorale qu'ils estiment devoir apporter à ceux qui solliciteraient d'eux un conseil et une ligne de conduite».

«N 88 . Dans le passé nos deux Communions ont développé chacune de leur côté leur doctrine morale et leur discipline pratique et pastorale. Les différences qui sont nées sont sérieuses, mais une étude et un examen attentifs ont montré qu'elles ne sont pas fondamentales. Lurgence du moment et la complexité de la condition humaine exigent à présent que tout soit mis en śuvre pour qu'on se réunisse afin de donner un témoignage commun et une orientation commune pour le bien être de l'humanité et pour le bien de toute la création", in Commission internationale anglicane-catholique, Anglicans et catholiques, approches de l'unité, les quatre documents de la Commision ARCIC II, présentation par Mgr G. Dáucourt, Commentaires par Mgr C. M. O'Connor et M. Santer, collection Documents d'Eglise, Paris, coéd. Bayard éditions, Centurion, Fleurus-Mame, Cerf, 2000, p. 119-120.

S'agissant du transsexualisme, l'Eglise épiscopalienne d'Angleterre a consacré religieusement un mariage entre une femme et un individu né de sexe féminin et devenu de sexe masculin depuis une opération médicale, selon Philippe Icard, Directeur du Centre de droit européen de la Faculté de Droit de Dijon, «Note: Un droit d'accès au mariage protégé par la Cour de justice des Communautés européennes», in Recueil Dalloz, N¹4, 2004, p. 979. 
Pour l'Union européenne, c'est à chacun des Etats membres de déterminer les conditions du mariage. Le Secrétaire d'Etat britannique, lors de l'affaire de l'arrêt de la CJCE du 7 janvier 2004 concernant un mariage avec un transsexue ${ }^{18}$ a affirmé que le mariage ne saurait se définir comme «l'union stable d'un certain caractère». Une telle formule ne rejoint pas le monde britannique pourtant si diversifié. En tout cas, l'état des personnes demeure une compétence exclusive des Etats.

Le juge communautaire doit s'appuyer sur les différents statuts précisés par les ordres juridiques étatiques. C'est pour cette raison juridique que le juge communautaire refuse d'assimiler ces trois modalités que sont le partenariat, le concubinage et le mariage. Le juge communautaire se réfère aux définitions données par les Etats membres.

Il existe une très grande diversité entre les régimes juridiques nationaux concernant la vie de couple au sens large du terme.

Les Etats membres sont encore incapables de s'accorder dans ce domaine à l'échelon de l'Union européenne. Le juge communautaire doit donc s'inspirer des droits internes nationaux en vigueur, tout en devant préserver les principes fondamentaux déjà établis à l'échelon communautaire. La CJCE constate qu'il existe une volonté des législateurs nationaux de fournir différents statuts pour les couples hétérosexuels, les couples non mariés et les compagnonnages unisexes. Le juge communautaire peut donc utiliser la méthode de «la référence aux droits des Etats membres», s'il ne parvient pas à déceler en droit communautaire des éléments suffisants.

Dans son arrêt du 7 janvier 2004 déjà cité, la Cour de justice des Communautés européennes, siégeant à Luxembourg, s'appuie sur l'arrêt Goodwin du 11 juillet 2002 de la Cour européenne des droits de l'homme et des libertés fondamentales siégeant à Strasbourg. Cette dernière élargit quelque peu la notion de mariage. Selon la Cour, il n'est pas certain que l'on puisse aujourd'hui continuer d'admettre que les termes impliquent que le sexe doive être déterminé selon des critères purement biologiques. La Cour en déduit

\footnotetext{
${ }^{18}$ La plupart des Etats membres de l'Union européenne expriment leur volonté de permettre aux transsexuels de se marier conformément à leur nouvelle identité sexuelle, in Recueil Dalloz, $N^{\circ} 14,2004$, p. 981.
} 
que le fait de retenir le sexe envisagé à la naissance pour contracter mariage constitue en l'espèce une limitation portant atteinte à la substance du droit de se marier ${ }^{19}$.

La Cour européenne de sauvegarde des droits de l'homme siégeant à Strasbourg se réfère à la Charte des droits fondamentaux de l'Union européenne adoptée à Nice en 2000. Quand, cette Charte seulement déclaratoire, accèdera-t-elle à la normativité juridique en droit de l'Union européenne? Ce qui avait été prévu au moyen de la procédure de son intégration au Traité constitutionnel de l'Union européenne, traité que chaque Etat membre était appelé depuis 2004 à recevoir, en principe. Or pour la Cour de Strasbourg, cette Charte de 2000 présente une conception plus large du mariage.

Dans son arrêt du 7 janvier 2004, la Cour de justice des communautés européennes de Luxembourg fait part de sa conscience d'une évolution des mœurs. Cependant, elle ne s'estime pas compétente pour élargir l'interprétation juridique du mariage, d'y inclure par exemple la cohabitation, le concubinage, d'assimiler à la notion de conjoint ou d'époux la situation de partenaire. Selon la CJCE, cet élargissement constitue une décision d'une telle importance qu'il revient aux Etats membres de l'Union européenne de recourir aux moyens de leurs législations respectives et de contribuer à une législation communautaire. Sans cette harmonisation, l'exclusivité de la compétente des Etats subsiste.

Le statut civil des personnes demeure une compétence étatique dans l'Union européenne à la fin de l'année 2004. Le 20 février 2007 par exemple en France, la plus haute juridiction civile, la Cour de cassation, a rendu deux arrêts refusant des demandes d'adoption simple formées par des compagnes de mères biologiques: l'adoption au sein d'un couple homosexuel vient d'être jugée illégale. La voie judiciaire de l'adoption est sans doute fermée.

Mais la compétence du droit communautaire commencerait à vouloir s'introduire dans ce domaine encore réservé. Philippe Icard dans sa note de 2004 , déjà citée ${ }^{20}$, avait écrit que le titre IV du projet de Traité constitutionnel de l'Union européenne de 2004, avait introduit une coopération juridique en matière civile. Le

${ }^{19}$ Point 41 de l'arrêt de la CJCE du 7 janvier 2004, in Recueil Dalloz, N ${ }^{\circ} 14,2004$, p. 982.

${ }^{20}$ Recueil Dalloz, N 14, 2004, p. 982. 
commentateur semblait regretter que la CJCE n'ait point pris appui sur les dispositifs du droit dérivé et sur ce projet de Traité constitutionnel de l'Union européenne, afin d'honorer la part qui revient déjà au droit communautaire, pour ce qui a trait au statut civil dans l'Union européenne. Toutefois, le chroniqueur reconnaissait que la CJCE se devait d'abord de respecter les législations nationales. En effet, si l'article 9 de la Charte de Nice n'introduit pas de référence au sexe pour reconnaitre le droit de se marier, cet article renvoie aux législations de chaque Etat membre de l'Union européenne la garantie de ce droit de se marier. Et il importe en effet de constater qu'une majorité de droits nationaux des Etats membres distinguent assez nettement les statuts de partenaire et le mariage. Je relève cette autre remarque du chroniqueur: l'article 21 de la Charte déclare intèrdite toute discrimination fondée notamment sur l'orientation sexuelle, je cite Philippe Icard. Ce dernier avait émis l'hypothèse selon laquelle «le refus d'accorder des mêmes droits aux partenaires qu'aux conjoints constitue une discrimination fondée sur l'orientation sexuelle».

Conclusions: enjeux éthiques et théologiques de l'adoption dans le cadre d'un compagnonnage homosexuel

Le 12 octobre 2004, Monsieur Dominique Perben, Garde des Sceaux et ministre de la Justice avait répondu à la question écrite déposée le 3 août précédent par $M$. le Député Thierry Mariani ${ }^{21}$. Le débat se ‘poursuit tant en France qu'à l'étranger, et cela plus que jamais en 2006-2007; en effet, plusieurs Etats de l'Union européenne ont légalisé récemment le pacte civil pour l'ouvrir à deux personnes soit du même sexe, soit de sexes différents; des Etats ayant légalisé parfois le principe du mariage homosexuel.

En éthicien et en théologien catholique, Xavier Lacroix critique les dérives d'une culture de la déliaison et de la dissociation entre conjugalité et parentalité. Il invite à découvrir une culture du lien, de la solidarité, de l'engagement. Quelle famille pour demain? Qu'est-ce qu'un père ${ }^{22}$ ?

${ }^{21} \mathrm{~N}^{\circ} 45117$, in $J O$, Assemblée nationale, Questions écrites, p. 7976.

${ }^{22}$ Xavier Lacroix, L'avenir, c'est l'autre, op. cit.; Id., "Sur le mariage homosexuel», in Etudes, décembre 2004, p. 613-623; O. Boulnois (Dir.), La différence sexuelle (Dossier), in Communio, tome XXXI 5-6, 2006, 136 pages; Jacques Arènes, «La problématique du 'genre'», in Documents épiscopat, N¹2,2006, 18 pages; Guy Bedouelle, Jean-Louis Bruguès, P. Becquart, L'Eglise et la sexualité, repères historiques et regards actuels, 
L'enfant à adopter tout comme l'enfant à engendrer ne doivent pas être les otages d'un désir, mais vivre leur vocation, où finalité et liberté vont apprendre non pas à s'épouser, mais à s'entendre.

\begin{abstract}
ANNEXE
Lannée 2000 était la première année de pleine application du PACS en France: 22276 PACS avaient été conclu. Il y en avait 40 093 en 2004; 60473 en 2005; 76302 en 2006. La fiscalité a été améliorée en janvier 2005: entre 2004 et 2005, la France a compté $50 \%$ de «pacsés» en plus. Depuis janvier 2007, les tribunaux d'instance sont autorisés, à la demande de l'Union européenne, d'enregistrer le sexe de chaque signataire. La hausse continue des PACS semble surtout due à la demande hétérosexuelle de couples en concubinage qui sont de plus intéressés par le PACS. Peut-être est-il possible de dire que le nombre de PACS homosexuels serait entre 10000 et 12000 . Si en 2006, il y a eu 268100 mariages civils hétérosexuels, cela représente néanmoins une baisse de 8000 mariages par rapport à l'année 2005. (cf. Marianne Gomez, «Le PACS poursuit sa progression», in La Croix, mardi 27 février 2007).
\end{abstract}

\title{
Wokół małżeństwa homoseksualnego? Prawo, etyka, teologia
}

Autor w swoim artykule omawia, w aspekcie prawnym, etycznym i teologicznym, kwestię małżeństwa zawieranego pomiędzy osobami tej samej płci. W tytule swego opracowania celowo stawia znak zapytania, sugerując opinię, czy w ogóle taki związek można nazywać małżeństwem, jak to czyni prawodawstwo niektórych państw europejskich. Analiza ustawodawstwa francuskiego prowadzi do stwierdzenia, że różnica płci jest nadal podstawą zawarcia małżeństwa we Francji. Możliwy jest jednak cywilny związek przyjaźni (pacte civil de solidarité$P A C S$ ) jako swoista forma konkubinatu. Niemniej jednak dwa kraje europejskie: Holandia (2000 r.) i Belgia (2003 r.) zmodyfikowaly swoje ustawodawstwo na korzyść małżeństwa homoseksualnego. Podobnie Kongres deputowanych Hiszpanii w 2005 r. przeglosowal prawo zezwalające na zawieranie takich małżeństw.

collection Histoire du christianisme, Paris, Cerf, 2006n 271 pages; Timothy Radcliffe op, «Les homosexuels peuvent-ils être prêtres?», in Revue d'éthique et de théologie morale, N²38, mars 2006, p. 113-116; René Heyer, La Condition sexuée, collection Etudes d'histoire ert de philosophie religieuse, Paris, PUF, 2006, 178 pages. 
Te zmiany i tendencje od strony etycznej i teologicznej zmuszają do postawienia poważnych pytań: jak ta nowa wizja rodziny ma się do rodziny tradycyjnej opartej na prawie naturalnym, czyli małżeństwie heteroseksualnym? Jaka jest jej przyszłość? Jaka jest przyszłość ojcostwa? Co w tym kontekście znaczy termin ojciec? Gdzie należy poszukiwać argumentów i odpowiedzi na rodzące siê pytania. Ponadto dziecko adoptowane w takim związku homoseksulanym nie może być zakładnikiem dwojga osób i ich pragnień seksualnych, lecz żyć swoim powołaniem i wolnością.

ks. Józef Wroceński SCJ 